\title{
A Single Oral Dose of Geranylgeranylacetone Upregulates Vascular Endothelial Growth Factor and Protects against Kainic Acid-Induced Neuronal Cell Death: Involvement of the Phosphatidylinositol-3 Kinase/Akt Pathway
}

\author{
Yukari Kawasaki Minoru Fujiki Susumu Uchida Masaki Morishige \\ Yasutomo Momii Keisuke Ishii \\ Department of Neurosurgery, School of Medicine, Oita University, Oita, Japan
}

\section{Keywords}

Kainic acid · Geranylgeranylacetone · Vascular endothelial growth factor . Phosphatidylinositol-3 kinase $\cdot$ PI3K/Akt pathway $\cdot$ Neuroprotection

\begin{abstract}
Background: Previous studies demonstrated the cytoprotective effect of geranylgeranylacetone (GGA), a heat shock protein inducer, against ischemic insult or kainic acid (KA)induced neuronal cell death. Phosphatidylinositol-3 kinase (PI3K)/Akt is thought to be an important factor that mediates neuroprotection. However, the signaling pathways in the brain in vivo after oral GGA administration remain unclear. Methods: We measured and compared hippocampal neuron density to investigate the effect of GGA on KA-induced cell death in rats. We evaluated the effects of pretreatment with wortmannin (Wort), a specific PI3K inhibitor, on GGAinduced neuroprotection against KA-induced cell death. To clarify the relationship between PI3K/Akt activation and neuroprotection, we used immunoblot analysis to deter-
\end{abstract}

() 2017 S. Karger AG, Basel mine the amounts of $\mathrm{p}$-Akt and vascular endothelial growth factor (VEGF) proteins present after GGA administration with or without Wort treatment. Results: Neuroprotective effects of GGA (pretreatment with a single oral dose of GGA, 800 $\mathrm{mg} / \mathrm{kg}$, $48 \mathrm{~h}$ before KA injection) were prevented by Wort pretreatment, which indicates that the selective PI3K/Akt pathway may mediate the GGA-dependent protection. Oral GGA-induced p-Akt and VEGF, and GGA pretreatment enhanced KA-induced VEGF, both of which were prevented by Wort pretreatment. Conclusion: These results suggest that a single oral dose of GGA induces p-Akt and that GGA plays an important role in neuroprotection against KA-induced neuronal cell death through VEGF induction.

๑) 2017 S. Karger AG, Basel

\section{Introduction}

Recent studies have demonstrated that a single oral dose of geranylgeranylacetone (GGA) is a convenient noninvasive pretreatment strategy for neuronal protec-

\section{KARGER}

E-Mail karger@karger.com

www.karger.com/pat
Minoru Fujiki

Department of Neurosurgery, School of Medicine

Oita University, 1-1, Idaigaoka, Hasama-machi

Oita 879-5593 (Japan)

E-Mail fujiki@oita-u.ac.jp 
Fig. 1. Experimental protocol. Time course group: animals prepared for Western blot analysis for the time course of HIF-1a, VEGF, VEGFR2, and PKCe expression after GGA administration. Another group of animals received a single oral dose of GGA, at varying $\mathrm{KA}$ injection intervals $(0,6,12$, $24,48,96$, and $192 \mathrm{~h}$ ) to assess the effect of the GGA-KA interval on neuroprotection. Control group: vehicle administration before intraperitoneal KA injection $(n=6)$. Second control group: $5 \mu \mathrm{L}$ in $100 \%$ DMSO i.c.v. $30 \mathrm{~min}$ before vehicle administration $(n=10$, i.e., $6+4)$. Wort group: vehicle administration with coinjection of Wort $(n=$ 10). GGA group: $800 \mathrm{mg} / \mathrm{kg}$ GGA pretreatment with coinjection of DMSO $(n=10)$. Wort-GGA group: GGA pretreatment with coinjection of Wort $(n=10)$. Animals were sacrificed $24 \mathrm{~h}$ after KA injection for HE staining, $3 \mathrm{~h}$ after oral administration ( $n=4$ in each group) or $1 \mathrm{~h}$ after KA injection ( $n=4$ in each group) for $\mathrm{p}$-Akt and $\mathrm{t}$ Akt Western blot analysis.

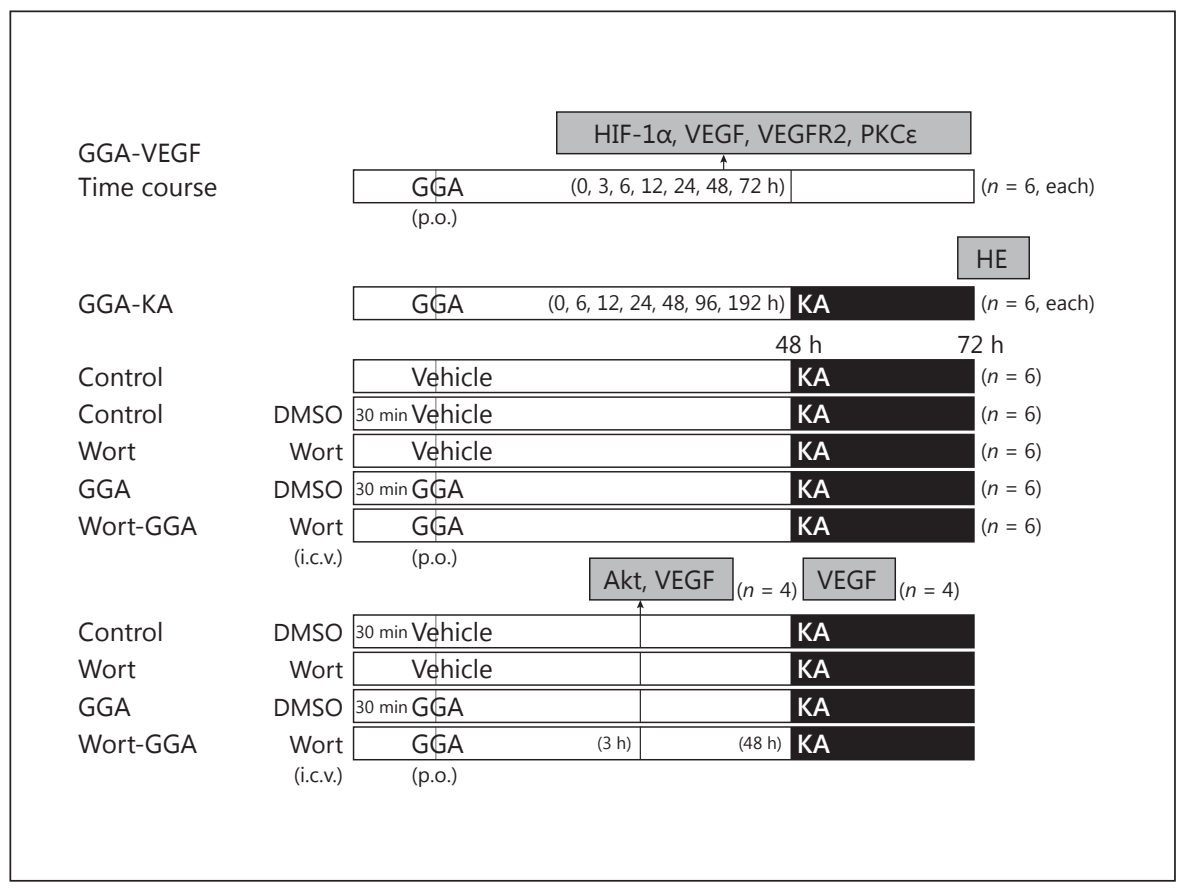

tion against ischemic insult and kainic acid (KA)-induced neuronal cell death $[1,2]$. However, the signaling pathways in the rat brain after GGA administration remain to be defined. Antiapoptotic activity of Akt is mediated by the activation of the phosphatidylinositol-3 kinase (PI3K)/ Akt pathway [3]. Vascular endothelial growth factor (VEGF) was reported to be involved in cerebral ischemic preconditioning [4]. There is strong support for the hypothesis that VEGF mediates neuroprotection via the activation of the PI3k/Akt pathway [4]. We postulated that the GGA-activated PI3K/Akt pathway is upstream of VEGF upregulation, and that activation of the latter leads to preservation of neuroprotective and neurological functions. In this study, we used a rat experimental model for neuronal cell death after KA injection to investigate the neuroprotective role of GGA. Specifically, we tested the hypothesis that oral GGA would induce p-Akt and, thereafter, VEGF upregulation would be involved in neuroprotection against neuronal cell death. This is the first report that investigates the role of the PI3K/Akt pathway and VEGF upregulation in the neuroprotective effects of GGA.

\section{Materials and Methods}

Animals and Experimental Protocol

All experimental protocols were approved by the Oita University Ethical Review Committee. Male Wistar rats (body weight
230-280 g) were housed at controlled room temperature (24.5$25.0^{\circ} \mathrm{C}$ ) with a 12 -h light/12-h dark cycle. The rats had free access to food pellets and tap water. GGA, an emulsion with $5 \%$ gum arabic and $0.008 \%$ tocopherol, was given orally before KA administration $(6 \mathrm{mg} / \mathrm{kg}$ i.p. dissolved in phosphate-buffered saline; Sigma, St. Louis, MO, USA; Fig. 1). The oral dose of $800 \mathrm{mg} / \mathrm{kg}$ was based on previous observations [5] showing that a single oral GGA dose $(800 \mathrm{mg} / \mathrm{kg}) 48 \mathrm{~h}$ before KA administration significantly attenuated hippocampal CA3-CA4 neuronal cell death.

In one experiment, animals received a single oral dose of GGA, and then the GGA-KA administration interval was varied $(0,6,12$, $24,48,96$, and $192 \mathrm{~h}$ before KA; $n=6$ at each interval). Rats in the sham vehicle-treated control group $(n=6)$ were given the vehicle with $5 \%$ gum arabic and $0.008 \%$ tocopherol $48 \mathrm{~h}$ before KA injection.

In a second experiment, the correlation with neuroprotection against KA administration was examined by coinjecting wortmannin (Wort; $16 \mu \mathrm{g} / \mathrm{kg}, 5 \mu \mathrm{L}$ in 100\% DMSO, using a 5- $\mu \mathrm{L}$ Hamilton syringe into the intracerebral lateral ventricle (i.c.v.), $30 \mathrm{~min}$ before 800 mg/kg GGA; Sigma), a specific PI3K inhibitor [6, 7].

Animals were classified into 5 groups for the second experiment: a control group (vehicle administration alone) and a second control group $(5 \mu \mathrm{L}$ in $100 \%$ DMSO i.c.v. 30 min before vehicle administration, $n=6$ each), a Wort group (vehicle administration with coinjection of Wort, $n=6$ ), a GGA group ( $800 \mathrm{mg} / \mathrm{kg}$ GGA pretreatment with coinjection of DMSO, $n=6$ ), and a Wort-GGA group (GGA pretreatment with coinjection of Wort, $n=6$ ).

Correlations with p-Akt and VEGF induction after GGA administration with or without KA were examined by coinjecting Wort (16 $\mu \mathrm{g} / \mathrm{kg}$, i.c.v., $30 \mathrm{~min}$ before $800 \mathrm{mg} / \mathrm{kg}$ GGA or vehicle). In this experiment, animals treated with KA were classified into 4 groups: a control group (pretreatment with DMSO before vehicle 
administration, $n=4$ ), a GGA group ( $800 \mathrm{mg} / \mathrm{kg}$ GGA pretreatment with coinjection of DMSO, $n=4$ ), a Wort-vehicle group (pretreatment with Wort before vehicle administration, $n=4$ ), and a Wort-GGA group (pretreatment with Wort before GGA administration, $n=4)$. Animals without KA were classified into 4 groups: a control group (pretreatment with DMSO before vehicle administration, $n=4)$, a GGA group ( $800 \mathrm{mg} / \mathrm{kg}$ GGA pretreatment with coinjection of DMSO, $n=4$ ), Wort-vehicle group (pretreatment with Wort before vehicle, $n=4$ ), and a Wort-GGA group (pretreatment with Wort before GGA administration, $n=4$ ).

For Western blot analysis, the animals without KA were sacrificed $3 \mathrm{~h}$ after oral administration ( $n=4$ in each group) or $1 \mathrm{~h}$ after KA and $48 \mathrm{~h}$ after oral GGA administration ( $n=4$ in each group). We accepted the time point $3 \mathrm{~h}$ after oral administration after reviewing data of the preliminary experiment $(2,3$, and $4 \mathrm{~h}$ after oral administration; data not shown). Separate sets of animals were prepared for Western blot analysis for the time course of hypoxiainducible factor (HIF)-1 $\alpha$, VEGF, VEGFR2 and protein kinase $\mathrm{C}$ $(\mathrm{PKC}) \varepsilon$ expression after GGA administration $(0,3,6,12,24,48$, and $72 \mathrm{~h}$ after GGA or vehicle, $n=4$, respectively).

\section{Histological Evaluation for Quantification of KA-Induced} Neuronal Cell Death

Rats were deeply anesthetized with sodium pentobarbital and perfused transcardially with $4 \%$ paraformaldehyde 3 days after the administration of GGA (1 day after KA injection). The $3-\mu \mathrm{m}$-thick paraffin-embedded coronal sections, including all of the hippocampal CA1-CA3 and CA4 regions, were stained with hematoxylin and eosin (HE). For each rat, an area of $1,500 \times 500 \mu \mathrm{m}$ that incorporated the CA3-CA4 region of the hippocampus [8] was counted in triplicate. Both sides of the brain were examined, and the average cell counts were taken. Only large intact cells with clearly stained cytoplasm and a distinct nucleus, usually with evident nucleoli, were counted as healthy neurons. Cells with shrunken cell bodies and nuclear condensation (apoptotic cells), or round cell bodies with pale cytoplasmic staining that showed signs of cytoplasmic vacuolation and fragmentation (necrotic cells), were counted as dead or dying cells.

Western Blot Analysis for Akt, HIF-1 $\alpha$, VEGF, VEGFR2, and $P K C \varepsilon$

For Western blot analysis, the left hemisphere was transferred to an ice-cold mammalian tissue lysis/extraction reagent (Sigma) that contained a protease inhibitor cocktail (Sigma). The tissues were then homogenized. The homogenate was centrifuged at $1,500 \mathrm{~g}$ for $10 \mathrm{~min}$ at $4^{\circ} \mathrm{C}$. The supernatant was stored at $-80^{\circ} \mathrm{C}$. Protein concentrations were determined by a previously described method [1] using bovine serum albumin as a standard (Bio-Rad). Equivalents of $40 \mu \mathrm{g}$ protein extracts from brain tissue were separated by sodium dodecyl sulfate-polyacrylamide gel electrophoresis. Protein separation was performed in $10 \%$ polyacrylamide with $0.05 \%$ bis-acrylamide. The proteins were then electrophoretically transferred to PVDF (polyvinylidene difluoride) membranes. After being transferred and blocked with $0.5 \%$ nonfat milk, the membranes were incubated with polyclonal antibodies (rabbit anti-Akt: 1:400 [Cell Signaling] and mouse anti-phospho-Akt: 1:400 [Cell Signaling]; mouse anti-HIF-1a: 1:200 [Santa Cruz Biotechnology, Santa Cruz, CA, USA]; anti-PKCe: 1:400 [Sigma-Aldrich, St. Louis, MO, USA]; rabbit anti-VEGF-A and VEGFR2: 1:400 [Santa Cruz Biotechnology]). Signals were detected by electrochemilumi- nescence with exposure to Hyperfilm (Amersham Pharmacia). Each blot was probed for total Akt or for $\beta$-actin (1:200; SigmaAldrich) as an internal control to ensure equivalent protein loading and protein integrity. The amount of protein on the immunoblots was quantified with NIH Image software.

\section{Statistical Analysis}

All data are represented as means \pm SEM. Different groups of animals were compared using the 2 -tailed Student $t$ test for independent pairs or 1-way (2-way for time course) ANOVA with the Student-Newman-Keul post hoc analysis (SPSS, Inc., Cary, NC, USA). Experiments with 3 or more groups were analyzed with 2-way ANOVA, followed by the post hoc Bonferroni-Dunn test. Differences were considered significant at the $p \leq 0.05$ level of probability.

\section{Results}

\section{Physiological Conditions}

No significant differences were found in body temperature and other physiological variables (blood $\mathrm{pH}$, blood gases, and hematocrit) between the groups. No differences in physiological variables were apparent between the groups with different GGA-KA intervals and those with different GGA concentrations (data not shown).

Time Course of Increases in HIF-1 $\alpha$, VEGF, VEGFR2, and $P K C \varepsilon$ Expression following GGA

Figure $2 \mathrm{a}, \mathrm{b}$ illustrates the time course of HIF-1a, VEGFR2, VEGF, and PKC $\varepsilon$ protein upregulation of animals sacrificed $3,6,12,24,48$, and $72 \mathrm{~h}$ after stimulation in rats treated with GGA. VEGF and VEGFR2 protein levels increased substantially $48 \mathrm{~h}$ after KA administration (treatment effect: $p=0.0003$ ). HIF- $1 \alpha$ and PKC $\varepsilon$ protein levels increased in a different manner (peak levels at $3 \mathrm{~h}$ in HIF-1a and PKCE, respectively), but this was not statistically significant. After the peak intervals, HIF-1 $\alpha$, VEGF, VEGFR2, and PKCe protein levels began to decrease, returning to near control levels by $72 \mathrm{~h}$ in HIF-1a and PKCe. At $72 \mathrm{~h}$, they were still higher in VEGF and VEGFR2 (Fig. 2a, b), and the differences were statistically not significant.

\section{Effect of Wort Pretreatment on GGA-Induced p-Akt and VEGF Expression}

One-way ANOVA performed on the p-Akt and VEGF expression showed main effects of treatment $\left(\mathrm{F}_{3,12}: 18.8\right.$, $p=0.0007)$. Specifically, $\mathrm{p}$-Akt and VEGF expression 3 and $48 \mathrm{~h}$ after treatment increased in the GGA group compared with the control group, respectively $(p<0.05$; Fig. 2c, e). Pretreatment with Wort before GGA sup-
186

Pathobiology 2017;84:184-191

DOI: $10.1159 / 000452862$
Kawasaki/Fujiki/Uchida/Morishige/ Momii/Ishii 

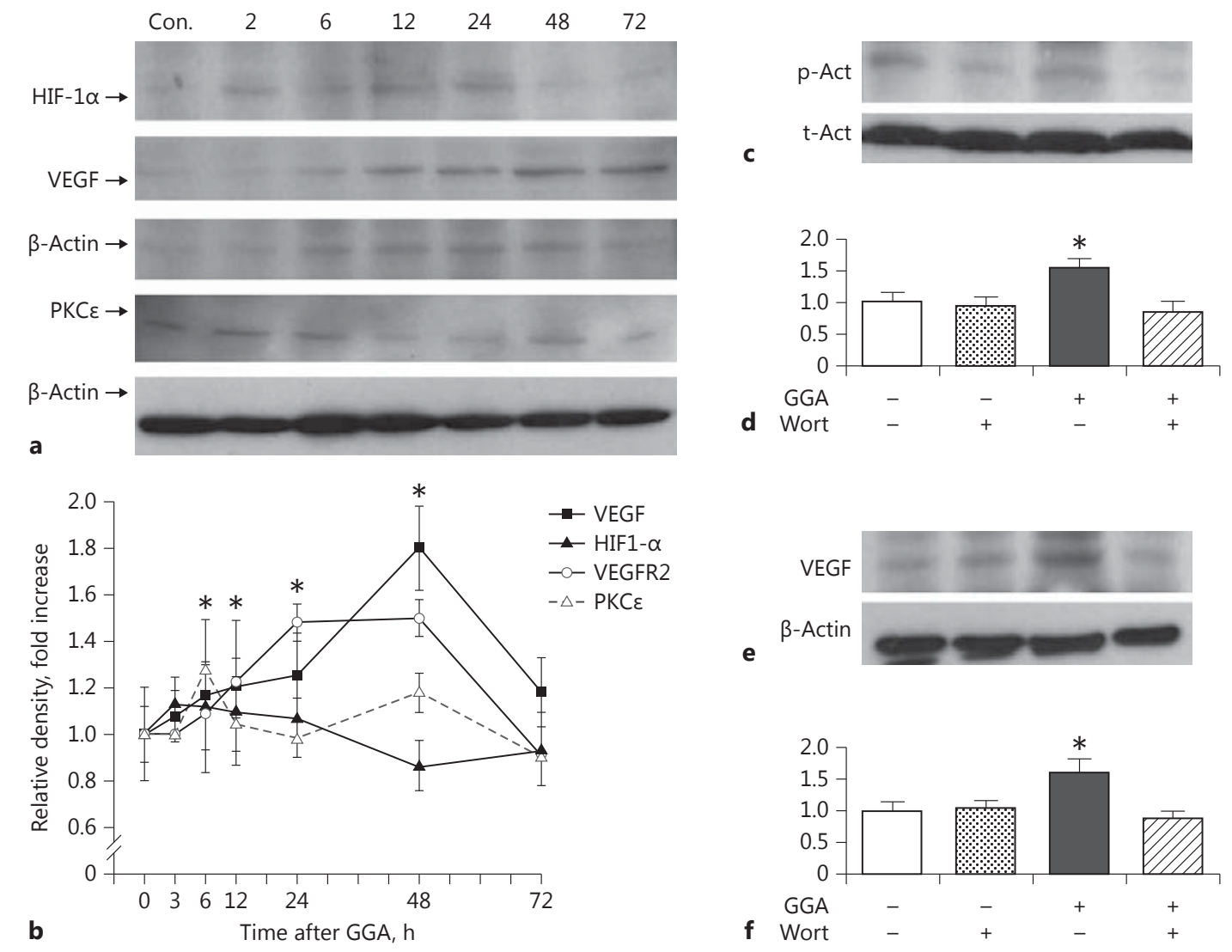

Fig. 2. Quantitative analyses of the increases in HIF-1a, VEGF, VEGFR2, PKCe, and p-Akt levels after GGA administration assessed by Western blot. a Representative bands of HIF-1a, VEGF, VEGFR2, PKC $\varepsilon$, and $\beta$-actin proteins 3, 6, 12, 24, 48, and $72 \mathrm{~h}$ after GGA administration. b Average relative density in the hippocampus of the left hemisphere $3,6,12,24,48$, and $72 \mathrm{~h}$ after GGA or vehicle administration. Means $\pm \operatorname{SEM}(n=4 /$ time point $) .{ }^{*} \mathrm{p}<0.05$

pressed GGA-induced p-Akt and VEGF expression, but pretreatment with Wort alone did not influence their expression. This resulted in no significant difference among the control, Wort-vehicle, and Wort-GGA groups (Fig. 2c-f).

\section{Neuroprotective Effect of GGA}

GGA significantly prevented neuronal cell death induced by KA injection in rats. As previously demonstrated, preservation of hippocampal CA3-CA4 neurons oc- vs. 0 h. c, e Effect of Wort pretreatment on GGA-induced p-Akt ( $3 \mathrm{~h}$ after administration; c) and VEGF expression (48 $\mathrm{h}$ after administration; e) assessed by Western blot in 4 groups (representative blots of $\mathrm{p}$-Akt and t-Akt as well as VEGF and $\beta$-actin expression are shown). d, $\mathbf{f}$ Quantitative evaluation of $\mathrm{p}$-Akt (d) and VEGF (f) expression in 4 groups. Means \pm SEM. $n=4$ /group. $* \mathrm{p}<0.05$ vs. control.

curred only when GGA was given $48 \mathrm{~h}$ before KA administration [5]. There was significant, but modest neuronal preservation when GGA was given for shorter or longer intervals than $48 \mathrm{~h}$ (Fig. 3a).

Coinjection of Wort with GGA abolished the protective effects, but Wort alone did not influence the protective effects on CA3-CA4 neuron survival (HE staining) $24 \mathrm{~h}$ after KA injection (Fig. 3b). 


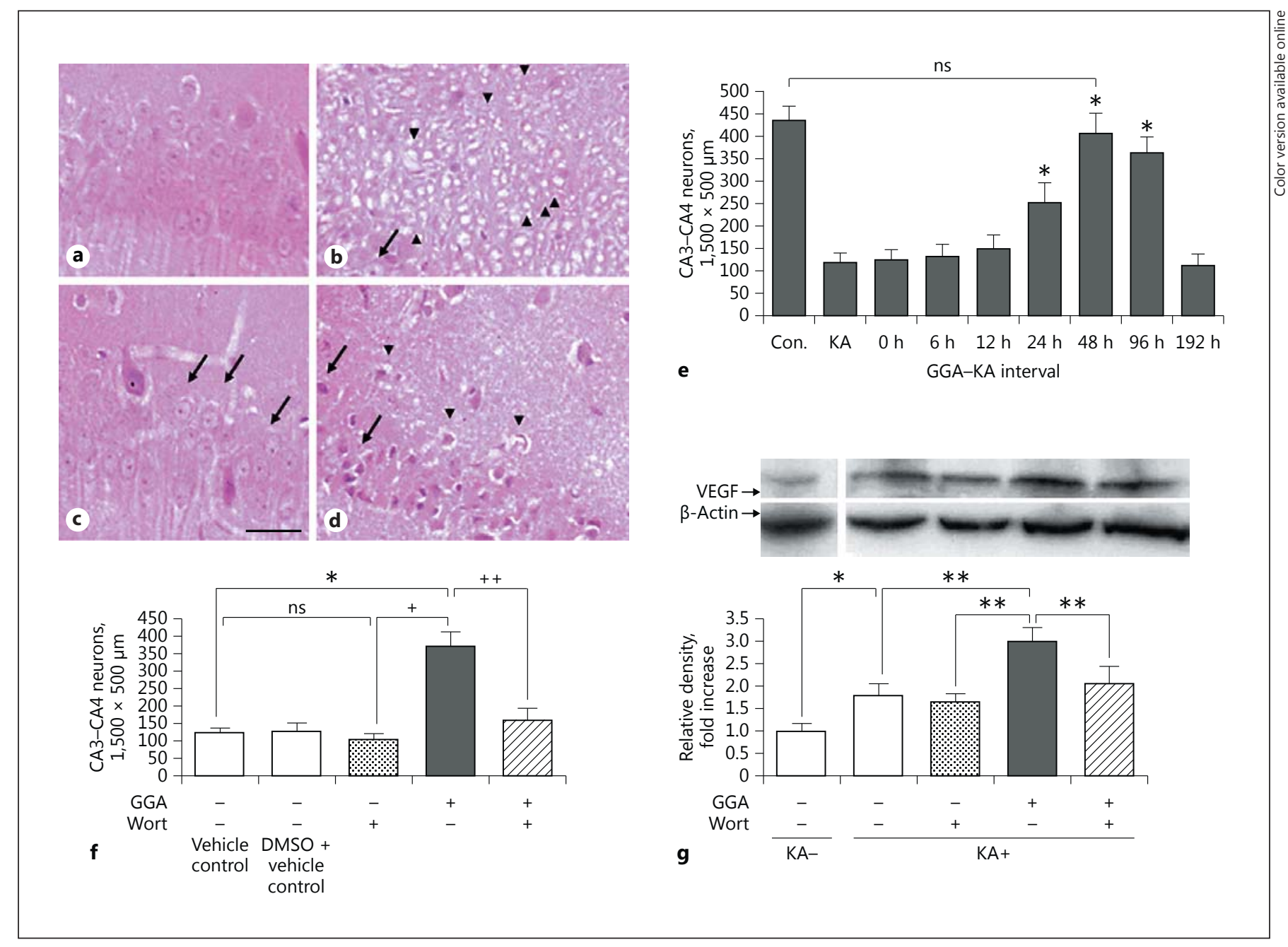

Fig. 3. Effect of GGA on neuronal cell death after KA administration. Photomicrographs indicating neuronal density in the CA3CA4 region of the hippocampus in a rat 1 day after KA injection (b) and in a rat with GGA preconditioning 2 days before KA (c): note the prominent cell loss in the CA3-CA4 area in the rat subjected to KA only (showing the transition between healthy cells [arrows] and dead or dying cells [arrowheads]; b) and the relative absence of neuron loss in the animal that had received GGA preconditioning (showing a mixture of some dying cells in healthy cells; c). a Cell density in a control case. $\mathbf{d}$ Abolished protective effect by showing an increased population of dying cells (arrowheads) after coinjection of Wort with GGA. Scale bar, $50 \mu \mathrm{m}$. e Effect of the GGA-KA interval on neuroprotection revealed by the number of surviving neuronal cells in HE-stained sections, which was significantly increased at the 48 -h interval. There was significant but modest neuronal preservation when GGA was given at shorter or longer intervals than the 48 -h interval. The graph shows the average neuronal cell counts in the hippocampal CA3CA 4 area $24 \mathrm{~h}$ after KA injection (6 animals/time point). Each number in the horizontal axis indicates the interval of GGA-KA. Means \pm SEM. ${ }^{*} p<0.05$ vs. vehicle control. $\mathbf{f}$ Effect of Wort treatment combined with GGA on neuronal cell death after KA administration. The graph shows the average hippocampal cell counts for animals given treatment with vehicle control, i.c.v. control (vehicle administration alone and $5 \mu \mathrm{L}$ in $100 \%$ DMSO i.c.v. 30 min before vehicle administration) Wort pretreatment group and GGA pretreatment group. Note the extensive neuronal death in the animals injected with KA alone and the reduction in neuronal death in the animals that received $800 \mathrm{mg} / \mathrm{kg}$ GGA as preconditioning. Coinjection of Wort with GGA abolished the protective effects, but Wort alone did not influence the protective effects in terms of cell survival ( $n=6$ /group). The graphs indicate mean values, and the bars indicate SEM for hippocampal neuronal cell counts. ${ }^{*} p<0.05$ vs. vehicle-treated control group with $\mathrm{KA}$ injection; ${ }^{+} p<0.05$ vs. Wort-treated group; ${ }^{++} p<0.05$ vs. GGA- and Wort-treated group. ns, not significant. g Quantitative analyses of the increases in VEGF levels after GGA administration by Western blot analysis. Top panel: effects of GGA pretreatment on KA-induced VEGF upregulation $1 \mathrm{~h}$ after KA. Representative blots of VEGF and $\beta$-actin upregulation by Western blot analysis in 5 groups. Lower panel: quantitative evaluation of VEGF upregulation in 5 groups revealed VEGF increase after KA injection. GGA substantially enhanced the KA-induced VEGF upregulation ( $n=4$ /group). Means \pm SEM. ${ }^{*} p<0.05$ vs. vehicle-treated control group with KA administration. ${ }^{* *} p<0.01$ vs. GGA-treated group with KA administration. 
Increases in KA-Induced VEGF Levels after GGA

Administration

GGA pretreatment enhanced KA-induced VEGF expression. Pretreatment with Wort either alone or combined with GGA given $48 \mathrm{~h}$ before KA did not influence KA-induced VEGF expression (Fig. 3c). This resulted in no significant difference among the KA-control, Wortvehicle, and Wort-GGA groups.

\section{Discussion}

The present results demonstrate that GGA alone induces a great deal of p-Akt and VEGF, and GGA pretreatment enhances KA-induced VEGF expression (measured by immunoblotting), and Wort, a specific PI3K inhibitor, shows a statistically insignificant protective tendency after KA-induced neuronal cell death. Previous studies have revealed that after preconditioning, p-Akt values peak 1-4 h after treatment and return to normal values within $24 \mathrm{~h}[6,7]$. Upregulation of neurotrophic proteins following conditioning (electrical stimulation) is blocked by protein synthesis inhibitors given 5 min before or up to $12 \mathrm{~h}$ after a single preconditioning [9]. The present results demonstrated that a single oral dose of GGA upregulates p-Akt expression $3 \mathrm{~h}$ after administration, which then returns to normal. The results of the present study also demonstrate that VEGF levels in the rat brain increase $48 \mathrm{~h}$ after a single oral dose of GGA. The HIF1- $\alpha$ increase was modest and not significantly affected by GGA. These findings are compatible with the results of recent studies that showed a correlation between VEGF upregulation induced by noninvasive preconditioning $[10,11]$.

Pretreatment with Wort before GGA administration substantially suppressed GGA-induced expression of pAkt and VEGF. If GGA-induced neuroprotective effects depend only on p-Akt and VEGF expression, the neuroprotective tendency should not be found in Wort-GGA animals. In line with the protection of CA3-CA4 neuronal cells, $1 \mathrm{~h}$ after KA injection, VEGF expression was enhanced by GGA administration. The neuroprotective effects in the GGA group were completely counteracted by pretreatment with Wort, suggesting that the PI3K/Akt pathway and VEGF expression have important roles even though the role of Akt phosphorylation remains controversial. Studies have shown that ischemic preconditioning protects the brain by induction of ischemic tolerance resulting from a sublethal ischemic insult accompanied by PI3K/Akt pathway activation [3]. The PI3K/Akt path-

Neuroprotective Effects of GGA way is a central mediator in signal transduction pathways involved in cell growth, cell survival, and metabolism. Akt phosphorylates caspase 9 at Ser-196, thereby blocking cytochrome c-mediated caspase 9 activation in vitro [12]. Akt may rescue cells from apoptosis by inhibiting the Bax-dependent apoptosis pathway through a forkhead box transcription factor [3].

It is important to clarify whether GGA activates PI3K/ Akt signaling, thereby indirectly mediating VEGF upregulation, or whether GGA mediates the VEGF receptor directly. Although the association between Akt phosphorylation and VEGF upregulation has not been well clarified, our observations may be explained by the hypothesis that Akt phosphorylation and subsequent activation/upregulation of VEGF play a part in the protective mechanisms when brains pretreated with GGA are subjected to lethal injuries. The important role of VEGF in neuronal death after pilocarpine-induced seizure has been reported [13]. An angiogenic signaling cascade in response to electrical stimulation-induced seizure or preconditioning has been elucidated [14] and provides additional support for a neurotrophic factor. Here, we focus on VEGF because the expression of this factor is induced in the brain by noninvasive preconditioning, is involved in progenitor cell migration, and is implicated in neuronal survival $[7,15]$.

There have been reports that VEGF is strongly upregulated in neurons and glia $24 \mathrm{~h}$ following neuronal injury from pilocarpine-induced status epileptics, and it has been established that VEGF has a neuroprotective potential against status epilepticus-induced neuronal loss [13]. In fact, the present results reveal that GGA alone induces a great deal of VEGF, and GGA pretreatment enhances VEGF expression (assessed by immunoblotting) suggesting that VEGF expression plays an important key role in the neuroprotection after KA-induced seizures and neuronal death. The molecular mechanisms underlying the neuroprotective effect of VEGF have not been elucidated yet. Evidence suggests that activation of the VEGF receptor VEGFR2, which is overexpressed by neurons following preconditioning, triggers the intracellular PI3K/Akt pathway and inhibits caspase- 3 activity to mediate cell survival [2].

However, given the results obtained by the PI3K inhibitor Wort, the present results provide evidence supporting the notion that Akt phosphorylation mediated VEGF upregulation, which is a downstream target of Akt. These are early steps in the development of GGA-induced preconditioning [4].

Pathobiology 2017;84:184-191

DOI: $10.1159 / 000452862$ 
GGA mediates and induces proteins such as PKC, Ras, cyclooxygenase-2 (Cox-2), and thioredoxin (which may act as an antioxidant), as well as p-Akt to inhibit cascades of apoptosis by Bad, procaspase 9, and Bax-independent pathway inactivation [16]. Activation of PKC modulates cell viability pathways, which either protect neuronal cells [14] or induce cell death. PKCa and PKCe activate ERKs and JNK and inhibit p38MAPK activation [14]. At the mitochondrial level, PKC $\varepsilon$ activity may help to regulate adenosine-induced $\mathrm{mK}^{+}{ }_{\text {ATP }}$ channels, maintaining energy and reducing calcium influx during ischemia. In our previous study, GGA-preconditioned animals tended to show moderate increases in $\mathrm{PKC} \varepsilon$, even though this effect was not statistically significant, and this suggests that GGA-induced PKC $\varepsilon$ confers protection against cerebral ischemia, in part by maintaining mitochondrial function via ERK activity and potentially by mediating the function of $\mathrm{mK}^{+}$ATP channels [1].

The regulation of Cox-2, which is induced by cytokines and growth factors, is interesting in this context because it is induced by fibroblast growth factor-2 and in turn induces VEGF [17]. Whether GGA plays a role in any of these conditions is not clear. The neuroprotective action of preconditioning (GGA-induced Akt activation) was blocked by Wort treatment. Upregulation of both VEGF and VEGF receptors after brain ischemia suggests that the upstream signaling underlying Akt activation in both sublethal and lethal ischemia is associated with the upregulation of VEGF signaling during ischemia [3]. The transcriptional activator, CREB, is phosphorylated by Akt both in vitro and in situ at Ser133 [18]. The phosphorylation of Ser-133 increases binding of CREB to CBP and enhances CREB-mediated transcription [18]. The role of CREB phosphorylation by Akt in survival signaling is still unclear, although evidence indicates that CREB regulates the expression of genes critical for survival, such as those encoding cytokines and BDNF [19]. Hence, if neuroprotective genes are directly induced by GGA ( $\mathrm{p}$-Akt and/or VEGF), the factors that mediate the induction remain to be defined. Any of these alterations in gene expression could help to prevent from cerebral infarction. p-Akt, VEGF, or other neuroprotective genes may promote neuron survival or mediate neuroprotection, but how they are induced by GGA or how the protective genes actually operate remains to be established.

\section{Acknowledgments}

This work was supported by a grant from the Japanese Science and Technology Agency and the Ministry of Education, Culture, Sports, Science, and Technology. The authors are grateful to Y. Hagimori for her technical assistance and to Eisai Co. Ltd. for the gift of GGA.

\section{Disclosure Statement}

The authors have no conflicts of interest to disclose.

\section{References}

1 Fujiki M, Hikawa T, Abe T, Uchida S, Morishige M, Sugita K, Kobayashi H: Role of protein kinase $\mathrm{C}$ in neuroprotective effect of geranylgeranylacetone, a noninvasive inducing agent of heat shock protein, on delayed neuronal death caused by transient ischemia in rats. J Neurotrauma 2006;23:1164-1178.

2 Jin KL, Mao XO, Nagayama T, Goldsmith PC, Greenberg DA: Induction of vascular endothelial growth factor receptors and phosphatidylinositol 3'-kinase/Akt signaling by global cerebral ischemia in the rat. Neuroscience 2000;100:713-717.

3 Yano S, Morioka M, Fukunaga K, Kawano T, Hara T, Kai Y, Hamada J, Miyamoto E, Ushio $\mathrm{Y}$ : Activation of Akt/protein kinase B contributes to induction of ischemic tolerance in the CA1 subfield of gerbil hippocampus. J Cereb Blood Flow Metab 2001;21:351-360.
4 Kilic E, Kilic U, Wang Y, Bassetti CL, Marti $\mathrm{HH}$, Hermann DM: The phosphatidylinositol-3 kinase/Akt pathway mediates VEGF's neuroprotective activity and induces blood brain barrier permeability after focal cerebral ischemia. FASEB J 2006;20:1185-1187.

5 Fujiki M, Kobayashi $\mathrm{H}$, Inoue R, Ishii $\mathrm{K}$ : A single oral dose of geranylgeranylacetone attenuates kainic acid-induced seizures and neuronal cell death in rat hippocampus. Brain Res 2004;1021:281-285.

6 Li L, Qu Y, Mao M, Xiong Y, Mu D: The involvement of phosphoinositid 3-kinase/Akt pathway in the activation of hypoxia-inducible factor-1 $a$ in the developing rat brain after hypoxia-ischemia. Brain Res 2008;1197:152158.
7 Yin W, Signore AP, Iwai M, Cao G, Gao Y, Johnnides MJ, Hickey RW, Chen J: Preconditioning suppresses inflammation in neonatal hypoxic ischemia via Akt activation. Stroke 2007;38:1017-1024.

8 Paxinos G, Watson C (eds): The Rat Brain in Stereotaxic Coordinates. London, Academic Press, 1996

9 Steward O, Kelley MS, Schauwecker PE: Signals that regulate astroglial gene expression: induction of GFAP mRNA following seizures or injury is blocked by protein synthesis inhibitors. Exp Neurol 1997;148:100-109.

10 Newton SS, Collier EF, Hunsberger J, Adams D, Terwilliger R, Selvanayagam E, Duman RS: Gene profile of electroconvulsive seizures: induction of neurotrophic and angiogenic factors. J Neurosci 2003;23:10841-10851. 
11 Warner-Schmidt JL, Duman RS: VEGF is an essential mediator of the neurogenic and behavioral actions of antidepressants. Proc Natl Acad Sci USA 2007;104:4647-4652.

12 Cardone MH, Roy N, Stennicke HR, Salvesen GS, Franke TF, Stanbridge E, Frisch S, Reed JC: Regulation of cell death protease caspase- 9 by phosphorylation. Science 1998;282: 1318-1321.

13 Nicoletti JN, Shah SK, McCloskey DP, Goodman JH, Elkady A, Atassi H, Hylton D, Rudge JS, Scharfman HE, Croll SD: Vascular endothelial growth factor is up-regulated after status epilepticus and protects against seizureinduced neuronal loss in hippocampus. Neuroscience 2008;151:232-241.
14 Maher P: How protein kinase C activation protects nerve cells from oxidative stress-induced cell death. J Neurosci 2001;21:29292938.

15 Altar CA, Laeng P, Jurata LW, Brockman JA, Lemire A, Bullard J, Bukhman YV, Young TA, Charles V, Palfreyman MG: Electroconvulsive seizures regulate gene expression of distinct neurotrophic signaling pathways. J Neurosci 2004;24:2667-2677.

16 Barone FC, White RF, Spera PA, Ellison J, Currie RW, Wang X, Feuerstein GZ: Ischemic preconditioning and brain tolerance: temporal histological and functional outcomes, protein synthesis requirement, and interleukin-1 receptor antagonist and early gene expression. Stroke 1998;29:1937-1950.
17 Majima M, Hayashi I, Muramatsu M, Katada J, Yamashina S, Katori M: Cyclo-oxygenase-2 enhances basic fibroblast growth factor-induced angiogenesis through induction of vascular endothelial growth factor in rat sponge implants. Br J Pharmacol 2000;130: 641-649.

18 Du K, Montminy M: CREB is a regulatory target for the protein kinase Akt/PKB. J Biol Chem 1998;273:32377-32379.

19 Tao X, Finkbeiner S, Arnold DB, Shaywitz AJ, Greenberg ME: $\mathrm{Ca}^{2+}$ influx regulates BDNF transcription by a CREB family transcription factor-dependent mechanism. Neuron 1998; 20:709-726. 